\title{
Historia Política de la Tecnología: una propuesta metodológica para la historia de la arquitectura escolar (Ciudad de México, 1880-1920) ${ }^{1}$
}

\author{
A Political History of Technology: \\ A Methodological Proposal for \\ the History of School Architecture \\ (Mexico City, 1880-1920)
}

\section{Carlos Ortega Ibarra \\ Universidad Pedagógica Nacional \\ Unidad 151, Toluca, México \\ carlosoi@unam.mx}

historiayliteraturaupn@gmail.com

\begin{abstract}
Los historiadores de la arquitectura, la salud y la educación han estudiado la arquitectura escolar desde distintas orientaciones metodológicas. La más reciente se refiere a la cultura material escolar, que contempla al edificio como parte del proceso educativo. Pero estas historiografías han pasado por alto la interacción entre los aspectos técnicos y políticos que hacen posible la materialidad de una escuela. En este artículo se sugiere, tras un breve recuento historiográfico, la Historia Política de la Tecnología como una metodología complementaria para comprender la arquitectura escolar en tanto acto técnico en el cual intervienen factores epistémicos y políticos. Para ello se recurre a los primeros reglamentos técnicos referentes al establecimiento de una escuela primaria y la construcción de las primeras escuelas tipo en la Ciudad de México, durante los años de 1880 a 1920.
\end{abstract}

Resumen

Palabras clave: Arquitectura escolar, Tecnología, Política.

\footnotetext{
${ }^{1}$ Una versión preliminar fue presentada en el ciclo 2014-2015 del Seminario Permanente de Historia de la Educación y la Investigación Científica, coordinado por las Dras. Ariadna Acevedo y Eugenia Roldán, en el Departamento de Investigaciones Educativas del Cinvestav. Agradezco los comentarios realizados por la Dra. María Eugenia Chaoul Pereyra, que sirvieron para mejorar este artículo, así como las sugerencias de los dictaminadores del mismo, que hicieron posible su publicación.
} 


\begin{abstract}
Historians of architecture, health and education have studied school architecture taking different methodological perspectives. The most recent is focused on material culture, including school buildings as part of the educational process. However, these historiographies have paid little attention to the intersection of technical and political aspects in the materiality of a school. After a brief historiographical account, this article proposes a Political History of Technology as a complementary approach to understanding school architecture as a technical act in which epistemic and political factors are involved. With this purpose in mind, this article examines the first technical regulations created for the establishment of a primany school, and the construction of the first 'model' schools in Mexico City during 1880-1920
\end{abstract}

Abstract

Keywords: School Architecture, Technology, Politics.

\title{
Introducción
}

Entre 1880 y 1920 tuvieron lugar en la Ciudad de México varias iniciativas de arquitectos, ingenieros, médicos, profesores y compañías constructoras para conformar una arquitectura escolar, con base en los principios de la higiene pedagógica y las nuevas técnicas de construcción. Estos proyectos, dirigidos a la administración del general Porfirio Díaz, adquirieron paulatinamente una forma legal, mediante el diseño de los primeros reglamentos técnicos para el establecimiento de escuelas, y se materializaron en la construcción de los prototipos de las escuelas primarias que el gobierno federal deseaba replicar para todo el país. Este acto técnico, conocido como arquitectura escolar, ha sido escasamente estudiado en México.

Para dilucidar el tema en este artículo hacemos, en primer lugar, un breve recuento historiográfico sobre la emergencia de la arquitectura escolar como objeto de investigación entre los historiadores de la arquitectura, de la salud y la educación en las décadas más recientes, siendo estos últimos quienes han contemplado la arquitectura -en tanto que se trata de un discurso educativo - como un factor de la cultura escolar. Para entender cómo surgió esta arquitectura especializada es necesario recurrir a otros conceptos. De esta forma exponemos, en segundo lugar, los rasgos principales de la Historia Política de la Tecnología como propuesta metodológica que nos ayuda a definir la arquitectura escolar en tanto acto técnico, proyectado por actores técnicos, como resultado de la interacción del conocimiento científico relativo a ella y el sistema político de un momento dado. Con el objetivo de esclarecer esta propuesta se aborda, finalmente, el caso de los primeros reglamentos técnicos para el establecimiento de escuelas y la construcción de los edificios escolares tipo en la Ciudad de México, entre 1880 y 1920. En su diseño y materialización intervinieron diversos actores técnicos, de acuerdo con los conocimientos disponibles en materia médica, pedagógica 
y constructiva, además de las condiciones políticas del Porfiriato. El propósito que anima a este artículo consiste en sugerir la Historia Política de la Tecnología como una metodología complementaria para estudiar las condiciones materiales del mundo escolar.

\section{Un tema emergente para la historia: la arquitectura escolar}

En naciones europeas como Francia, España, Alemania e Inglaterra la arquitectura escolar se consolidó desde 1980 como tema de investigación para los historiadores del arte, la medicina, la arquitectura, la educación y las ciudades (Châtelet, 2004), mientras que en el caso latinoamericano el interés por esta cuestión es reciente. Al concluir la década de 1990 los arquitectos e historiadores de la salud y la educación se interesaron por la arquitectura escolar colonial o republicana de sus respectivos países de origen. En los casos argentino y colombiano ha predominado el interés por contribuir al conocimiento del patrimonio arquitectónico local, mediante la elaboración de inventarios o recuentos históricos de los principales edificios escolares, construidos por los regímenes republicanos de los siglos XIX al XX. En este sentido, las obras de los arquitectos Daniel Schávelzon (1989), Gustavo Brandariz (1997) y Rafael Maldonado Tapias (1999) hicieron visibles las arquitecturas escolares de ambos países. Posteriormente, Lucía Espinoza (2005) y Francisco Ramírez Potes (2009) reconocieron la importancia de incorporar aspectos políticos y educativos en los estudios históricos de la arquitectura escolar, recurriendo a los ejemplos de las ciudades de Santa Fe y Bogotá en el siglo Xx. Sin embargo, el enfoque arquitectónico no dio pie al desarrollo de la arquitectura escolar como tema de investigación histórica -con objetivos y metodología propios- en Colombia ni en Argentina.

En el caso de Brasil la amplia literatura sobre la cuestión nos muestra el desarrollo de una perspectiva que reconoce en los edificios escolares un discurso pedagógico y una función educativa. La arquitectura escolar dejó de ser exclusivamente una fuente de información documental para convertirse en objeto de estudio de los historiadores de la educación, como lo señalaron Rosa Fátima de Souza (1998) y Luciano Mendes de Faria (1998).

En los últimos años, Marcus Bencostta (2005) ha sido el principal promotor de los estudios históricos de la arquitectura escolar brasileña, quien la considera como transmisora de mensajes con sentidos múltiples. Sus esfuerzos por conformar un campo de estudio dieron lugar a la organización del Nucleo de Estudos e Pesquisas em História da Arquitectura Escolar (NEPHArqE), en la Universidad Federal de Paraná, cuyo principal objetivo es contribuir a la formación de investigadores especializados en la historia de la arquitectura y el espacio escolar. Los trabajos del centro se distinguen por la pluralidad metodológica con que se aborda este 
aspecto en distintas regiones del Brasil y su interés por conocer las experiencias de investigación en otros lugares de América Latina.

En México, la cuestión ha sido estudiada por arquitectos e historiadores, tanto de la salud como de la educación, de una manera similar a las de sus pares latinoamericanos. Los primeros -entre quienes encontramos a Víctor Arias (2005) y Axel Arañó (2011) - se dieron a la tarea de elaborar recuentos o inventarios históricos con el objetivo de incorporar los edificios escolares al legado arquitectónico nacional y conmemorar la obra constructiva de algún arquitecto, una institución pública o una época. La mayoría de estos trabajos se ciñe a la descripción de los "grandes proyectos arquitectónicos", sin considerar los aspectos socio-culturales o técnico-políticos que hicieron posible su conformación, salvo en los textos de Gerardo Sánchez (2005) y Natalia de la Rosa (2011), quienes destacaron los elementos técnicos y los compromisos políticos que dieron origen a la arquitectura escolar posrevolucionaria. Una mención aparte merece la obra del arquitecto Ramón Vargas Salguero (1998), para quien este tipo de edificios constituyen espacios producidos socialmente para la habitabilidad.

En contraste con el trabajo de la mayoría de los arquitectos, en los últimos años, algunas historiadoras de la salud y la educación han explorado nuevas vetas con resultados interesantes. Ana María Carrillo (1999 y 2010) y Anne Staples (2008) han dedicado parte de su obra a las políticas sanitarias que incidieron, a través de la conformación de la higiene pedagógica, en el establecimiento de escuelas públicas en el siglo XIX. Por otra parte, María Eugenia Chaoul Pereyra (2005) definió el espacio escolar de la Ciudad de México, durante el periodo porfiriano, como un espacio diferenciado o ambiente especializado, resultado de las relaciones sociales gestadas al interior de una escuela, las cuales determinan tanto el significado como el uso de un inmueble. Dichas autoras coinciden en apuntar que la arquitectura escolar cumple con una función dentro del proceso educativo, que está condicionada por la acción de los sujetos a quienes se dirige.

La historiografía nos muestra la pluralidad de enfoques que existen sobre el tema. Es necesario reconocer que los arquitectos hicieron visibles los edificios escolares al momento de colocarlos como objeto de estudio de la historia de la arquitectura y que otros historiadores, con un enfoque social o cultural, dedicados al estudio de la arquitectura, la salud y la educación, superaron la elaboración de los recuentos o inventarios arquitectónicos al ocuparse de los aspectos técnico, habitacional, sanitario y educativo de un local escolar. Sin embargo, en nuestro contexto, la arquitectura escolar es un campo de investigación histórica en ciernes. 


\section{La arquitectura como factor de la cultura escolar}

Los trabajos recientes en Brasil y México muestran la emergencia de la cultura material escolar como una nueva orientación teórico-metodológica de los historiadores de la educación, enfocados en el estudio de la arquitectura escolar. Consideremos algunos ejemplos.

Marcus Bencostta y Rosa Fátima de Souza coordinaron un número especial de Educar em Revista (2013) dedicado al tema. En la presentación del número señalan, parafraseando a Marie Pierre Julien y Céline Rosselin, autores de La Culture Matérielle, que la cultura material escolar consiste en la relación que los sujetos establecen con los objetos. Los primeros construyen una serie de significados, gracias a un proceso activo de percepción de los objetos, en tanto que los segundos ejercen funciones sociales, estéticas y simbólicas que inciden en los sujetos. La materialidad de la escuela -constituida, entre otros elementos, por el edificio, mobiliario, uniforme y utillaje escolares- adquiere múltiples significados en función de los fines sociales y culturales de un proceso educativo que tiene lugar en condiciones históricas específicas.

Uno de los artículos publicados en este número, "La intervención del Estado, la desigualdad y el deseo en la invención de los espacios escolares rurales en tres regiones de México en el periodo posrevolucionario", de Oresta López, Norma Ramos y Armando René Espinosa, se propone hacer una lectura etnográfica en torno a la cultura material de la escuela rural mexicana. Para ello retoman los casos de las escuelas fundadas en el valle del Mezquital (Hidalgo), Nuevo León y San Luis Potosí, entre 1920 y 1930. Al respecto, los autores consideran importante "leer al mundo escolar para describir e interpretar los múltiples significados que se tejen al interior de los espacios escolares" (López, Ramos y Espinosa, 2013: 61) y que condicionan su uso.

En consonancia con estos planteamientos, Rosalía Meníndez publicó Las escuelas primarias de la Ciudad de México en la modernidad porfiriana, en el que describe y analiza -con base en las sugerencias metodológicas de Agustín Escolano y Antonio Viñao- la cultura escolar conformada por aspectos formales como son los métodos, programas, horarios, tiempos, espacios y "otros rasgos íntimos de la vida de la escuela" (Meníndez, 2013: 29). Bajo este enfoque dedicó un capítulo de su libro a la arquitectura escolar, configurada por la higiene pedagógica al concluir el siglo XIX.

Cada uno de los autores mencionados reconoce que la cultura material escolar es un concepto complejo, cuestionado y de difícil estructuración teórico-metodológica. Aunque el nuevo enfoque hizo visible la materialidad de la escuela para los historiadores, hasta el momento ha dejado de lado algunas preguntas fundamentales, entre ellas: ¿cómo un objeto escolar, y de manera específica un edificio destinado a la enseñanza, llegó a ocupar el lugar 
específico en que se encuentra? y ¿cuáles fueron los factores epistémicos y no epistémicos que, mediante su interacción, hicieron posible su materialidad?

\section{La arquitectura escolar como un acto técnico}

Una respuesta a tales cuestionamientos implica la elaboración de una propuesta metodológica complementaria para la historia de la arquitectura escolar. La metodología de la Historia Política de la Tecnología, desarrollada a partir del estudio de casos en el Seminario de Historia de la Ciencia y la Tecnología en México - dirigido por el doctor Juan José Saldaña, en la Facultad de Filosofía y Letras de la UNAM- nos permite comprender la arquitectura escolar como un acto técnico para la construcción de inmuebles destinados a la enseñanza. En su edificación intervinieron factores epistémicos, como la sistematización y codificación del conocimiento médico, pedagógico y constructivo, en los reglamentos técnicos, por ejemplo, y factores no epistémicos, como los diversos intereses de los actores involucrados en el diseño y construcción de un edificio escolar. Veamos ahora de manera general algunos conceptos de esta metodología, como son: acto técnico, actores técnicos y sistema político, teniendo presente el caso de la arquitectura escolar de la Ciudad de México entre 1880 y 1920, que desarrollaremos más adelante.

Saldaña (2011) apunta que la tecnología, definida por Ruy Gama (1985) como la ciencia o logos de la producción, es un conocimiento con una dimensión sociopolítica que se consume en la acción y para la acción. Con esto nos remite a los "actos técnicos" de los que José Ortega y Gasset habla en su Meditación de la Técnica, en los cuales dedicamos nuestro esfuerzo a inventar y ejecutar un plan de acción, con el fin de asegurar la satisfacción de nuestras necesidades -empezando por las elementales- mediante el mínimo esfuerzo, además de crear "posibilidades nuevas produciendo objetos que no hay en la naturaleza del hombre" (Ortega, 1965: 34).

Bajo esta mirada, la arquitectura escolar es entendida como un acto técnico proyectado por actores técnicos en circunstancias históricas particulares. En el caso que nos interesa, los ingenieros, arquitectos, médicos y pedagogos formados en las escuelas superiores del Porfiriato, -una minoría cuyos proyectos estuvieron orientados por un orden institucional, un marco ideológico y las técnicas y conocimientos disponibles (Kuntz y Connolly, 1999)_² intentaron incidir mediante sus proyectos técnicos en la sistematización de los conocimientos

\footnotetext{
2 Sandra Kuntz y Priscila Connolly señalan que las obras públicas están condicionadas por un orden institucional, un marco ideológico y las técnicas y conocimientos disponibles. El conocimiento, el saber hacer y las relaciones establecidas con el poder político son recursos de que dispone un actor técnico. En un sentido más amplio,
} 
relativos a la construcción de escuelas y, en consecuencia, en el diseño de las políticas seguidas por el Estado mexicano sobre la materia, entre las últimas décadas del siglo XIX y la primera del Xx.2 Su actuación, apelando a la intervención del poder público a favor de sus iniciativas, nos permite identificarlos simultáneamente como actores políticos.

Para entender esto es necesario considerar que sus acciones se ciñeron a un sistema político constituido alrededor del poder Ejecutivo Federal, que actuó como articulador o concertador de los intereses involucrados, puesto que otros actores, quienes no necesariamente poseían una formación técnica-científica análoga a la de los profesionales aludidos, deseaban participar en la conformación del acto técnico, en función de sus intereses y cultura técnica específicos. Me refiero, por ejemplo, a compañías deslindadoras y colonizadoras de terrenos, empresas especializadas en la construcción de obras públicas (contratistas) o propietarios de inmuebles, quienes también esperaban obtener una respuesta satisfactoria a sus demandas, dirigidas tanto a la administración de Porfirio Díaz como a las que siguieron a su defenestración en 1911. En este sentido, cabe aclarar que los niños, a quienes principalmente estuvo dirigido el acto técnico de la arquitectura escolar, no son considerados como un actor técnico o político que incidiera en la conformación de la misma, lo que no quiere decir que sus acciones carecieran de repercusión en la configuración del espacio escolar, la construcción de significados sobre los lugares y el empleo de las instalaciones. ${ }^{3}$

Por su parte, Luis Medina Peña (2004) define el sistema político como el conjunto de reglas formales e informales (implícitamente acordadas) que rigen las relaciones entre los actores políticos y considera el Porfiriato como el primer sistema político "realmente operativo" en el que la figura del presidente de la República constituyó el nodo de una extensa red de relaciones políticas. La operatividad de este sistema radicó en la negociación, entendida como la base de la acción política, y su crisis, al concluir la primera década del siglo Xx, fue resultado de su incapacidad para incorporar en la negociación de los asuntos públicos a los actores que emergieron como resultado del proceso de modernización de la vida social. Entre ellos se encuentra un contingente significativo de científicos y técnicos que optaron por la formación de un nuevo orden político -emanado de un proceso revolucionario-, que hiciera posible la materialización de sus proyectos, incluyendo los relativos a la arquitectura escolar. Cabe mencionar que el estudio de los actores técnicos resulta necesario para

\footnotetext{
Jaime Fisher (2010) menciona que la técnica es el resultado de una transacción que involucra otros aspectos, como son los valores y las creencias de los actores.

${ }^{3}$ Es pertinente mencionar que los autores de los proyectos técnicos para la construcción de escuelas primarias concebían a los niños como una población heterogénea a la que era necesario introducir a la vida social moderna, salvaguardándola de los perjuicios generados por ella, mediante la escuela (Donzelot, 2008). El inmueble escolar tenía una función educativa, puesto que, en consonancia con los programas escolares, contribuía con la formación de nuevos hábitos en la población escolar, a partir de la organización de sus actividades por tiempos y lugares. En este sentido el niño era imaginado por los actores técnicos como un actor en formación, como un futuro obrero y soldado de la patria (Carrillo, 2005 y 2010).
} 
conocer el proceso histórico de la modernidad de la que fueron causa y efecto, en el que la escuela pública adquirió, con el tiempo, un papel relevante como inoculadora de sus valores entre la población de la Ciudad de México.

Finalmente, el acto técnico denominado como arquitectura escolar conjugó factores epistémicos y no epistémicos, pues como lo afirmaron Teresa Rojas Rabiela, José Luis Martínez y Daniel Murillo (2009) al referirse a la construcción de obras hidráulicas en Mesoamérica durante la época prehispánica: los sistemas sociopolíticos, junto con las técnicas, "constituyen la tecnología propiamente dicha." Sin ella el arte de gobernar -entendido también como un acto técnico - es impracticable en el mundo moderno.

\section{La arquitectura escolar de la Ciudad de México}

Al iniciar el siglo xx, 65 por ciento de los 720 mil habitantes del Distrito Federal (224 mil eran menores de 15 años) vivían en la municipalidad de México. Los museos, bibliotecas, escuelas y sociedades científicas se concentraban en esta entidad más que en cualquier otra del país, lo mismo que los profesores, médicos, abogados y hombres de armas (ejército, policía y marina). Sin embargo, ellos formaron una minoría intelectual en comparación con la mayoría de los habitantes del Distrito Federal, quienes eran analfabetas. Al concluir la primera década del siglo xx, la tasa de analfabetismo era de 77 por ciento (Estadísticas, 1956). Ello corrobora la afirmación de Mílada Bazant relativa a que la política educativa modernizadora porfiriana se dirigió principalmente a la formación de una "aristocracia del talento" (2000: 217), que tendría en sus manos la dirección de la República Mexicana; una minoría que imaginó a la Ciudad de México, cuya superficie se ampliaba al iniciar el siglo XX, como el faro irradiador de la modernidad.

Durante las décadas de 1880 a 1910 la Ciudad de México experimentó un proceso que algunos urbanistas llamaron "expansión de la ciudad" (Fernández, 2000), como consecuencia de políticas liberalizadoras que favorecieron la especulación de la propiedad territorial, inversión de capital en bienes raíces, migración de recursos humanos, colonización de las zonas rurales y construcción de obras de infraestructura urbana. Las estadísticas sociales de la época nos brindan algunas cifras de este proceso, pues en el Distrito Federal el número de casas en construcción pasó de 781 en 1895 a 1035 en 1900 (Estadísticas, 1956). Este proceso fue particularmente visible en el poniente, con la emergencia de nuevas colonias como la Guerrero, Santa María, San Cosme y San Rafael, entre otras.

La expansión de la ciudad, impulsada por compañías constructoras y deslindadoras de terrenos, implicó, entre otras cosas, la demanda de servicios como el alumbrado público, 
transporte, drenaje, agua potable y pavimentación de calles para las colonias recientemente fundadas, así como el aumento de la densidad de población en distintas zonas de la ciudad que carecían de infraestructura urbana. La capacidad del ayuntamiento de la Ciudad de México para atender este tipo de demandas era limitada. De ahí que, como plantea Ariel Rodríguez Kuri (1999), concesionara a empresas particulares la provisión de servicios como el del alumbrado público. ${ }^{4}$ De acuerdo con esta dinámica, tanto el ayuntamiento como el gobierno federal debieron recurrir al arrendamiento de casas de particulares para poder brindar instrucción primaria a la población citadina.

En el año de 1896 el ayuntamiento de la Ciudad de México, que había fungido como un espacio de representación de los actores locales, llegó a sostener 113 escuelas primarias para 13 mil alumnos, aproximadamente (véase cuadro 1). La mayoría se encontraba en casas arrendadas a particulares en malas condiciones de higiene y sus constantes reparaciones afectaban las arcas municipales (Pérez, 1883; Ruiz, 1900). ${ }^{5}$ Era una práctica común que los propietarios de los inmuebles demandaran a las autoridades educativas de la ciudad que pagaran el costo de la adaptación de las casas para ser utilizadas como escuelas y las reparaciones a que hubiere lugar por el uso del inmueble. ${ }^{6}$

Al concluir el siglo XIX el ayuntamiento llegó a ser propietario únicamente de tres fincas que fueron modificadas para que pudieran funcionar como escuelas primarias (Pérez, 1883; Ruiz, 1900). En estas condiciones, la Ciudad de México fue objeto de numerosas iniciativas, como la del señor Faustino Martínez, representante de una compañía deslindadora y colonizadora de terrenos, ${ }^{7}$ para implantar una arquitectura escolar que permitiera superar los problemas ocasionados por el arrendamiento de locales insalubres e inadecuados para el proceso educativo. No obstante se carecía de un marco legal que normalizara las características técnicas de un edificio escolar público.

Los primeros parámetros técnicos para la conformación de un modelo de edificio escolar fueron establecidos en sesiones prolongadas por los participantes de los congresos de

\footnotetext{
${ }^{4}$ Kuri sostiene que el hecho de concesionar un servicio a una empresa particular (el alumbrado público) y que el ayuntamiento se hiciera cargo de la administración de otro (el agua potable), se debió a la fuerza de los actores involucrados (1999).

${ }^{5}$ Desde 1867, la Comisión de Instrucción Pública del ayuntamiento de la Ciudad de México fue la instancia encargada de costear el establecimiento de las escuelas municipales. Los regidores Rafael Pérez Gallardo, Nicolás Ramírez de Arellano (médico) y Luis E. Ruiz (médico), quienes participaron en los congresos nacionales de higiene y pedagogía de la década de 1880, encabezaron dicha comisión entre 1882 y 1896, cuando se concretó la federalización de las escuelas municipales.

${ }^{6}$ De ello dan fe las solicitudes de los propietarios para que el ayuntamiento modificara las cláusulas de los contratos de arrendamiento, los cuales se encuentran en la Sección de Instrucción Pública del Fondo Ayuntamiento del Archivo Histórico del Distrito Federal (AHDF).

${ }^{7}$ En 1891 Faustino Martínez propuso al ayuntamiento de la Ciudad de México la construcción de veinte escuelas municipales mixtas por un costo aproximado de $\$ 1400000$, como una medida para resolver los problemas relativos al arrendamiento de casas y las indemnizaciones a los propietarios (Martínez, 1891).
} 


\section{Cuadro 1}

\section{Escuelas sostenidas por el ayuntamiento de la Ciudad de México, 1882-1896}

\begin{tabular}{|c|c|c|}
\hline Año & $\begin{array}{l}\text { Número de } \\
\text { escuelas }\end{array}$ & Tipo de escuelas \\
\hline & & 30 para la enseñanza elemental \\
\hline & & (15 para niños y 15 para niñas) \\
\hline \multirow[t]{7}{*}{1882} & 81 & 49 para la instrucción primaria \\
\hline & & (29 para niñas y 20 para niños) \\
\hline & & 1 para párvulos \\
\hline & & 1 para obreros \\
\hline & & 42 para niñas \\
\hline & & 34 para niños \\
\hline & & 4 mixtas \\
\hline \multirow[t]{5}{*}{1888} & 93 & 4 para párvulos \\
\hline & & 6 para obreros \\
\hline & & 3 para obreras \\
\hline & & 50 para niños \\
\hline & & 49 para niñas \\
\hline \multirow[t]{2}{*}{1896} & 113 & 5 mixtas \\
\hline & & 9 nocturnas suplementarias para adultos \\
\hline
\end{tabular}

Fuentes: Gallardo (1883) y Ruiz (1900).

Higiene Escolar de 1882 e Instrucción Pública de 1889-1891, patrocinados por la Secretaría de Gobernación (a través del Consejo Superior de Salubridad, dirigido por el médico Ildefonso Velasco) y la Secretaría de Justicia e Instrucción Pública, encabezada por el abogado Joaquín Baranda. En ambas reuniones, celebradas en la Ciudad de México, las comisiones encargadas de redactar los dictámenes sobre las características higiénico-pedagógicas de un inmueble escolar estuvieron encabezadas por quienes se apuntalaron como especialistas en la materia: el médico Domingo Orvañanos, el profesor Manuel Cervantes Imaz y el arquitecto-ingeniero Manuel Francisco Álvarez, entre otros, llegando los dos últimos a ser funcionarios del ramo de la instrucción pública en el Distrito Federal.

En dichos congresos se reconoció la incapacidad financiera de la administración pública local para construir edificios escolares, por lo que los dictámenes aprobados en ambas asambleas establecieron una serie de condiciones que las casas utilizadas como escuelas 
debían satisfacer, relativas a la localización y orientación del inmueble; dimensión, iluminación y ventilación de las salas de clase y características de los sanitarios e instalaciones anexas (gimnasios, talleres, patios de juego y habitaciones del profesor). Se pedía, por ejemplo, que las escuelas se establecieran sobre terrenos impermeables, natural o artificialmente, alejados de instalaciones insalubres como cementerios y fábricas, con una orientación que permitiera el aprovechamiento de la luz solar para los trabajos escolares a lo largo del día. Al respecto, los congresistas acordaron que la iluminación de las salas de clase fuera natural, unilateral izquierda o bilateral diferencial ("Actas", 1882; "Dictamen", 1890; "Congreso", 1890)

Los congresistas esperaban que sus resolutivos adquirieran una forma legal que orientara la acción de la sociedad para el establecimiento de escuelas en condiciones salubres, de acuerdo con una pedagogía que consideraba los inmuebles como elemento complementario para el desarrollo intelectual y físico de los niños a través del juego y el trabajo sistematizados, según las doctrinas pedagógicas de Enrique Pestalozzi y Federico Froebel. Los resolutivos adquirieron una forma legal incipiente en la década de 1890, cuando el Poder Ejecutivo federal, encabezado por el general Porfirio Díaz, se propuso modernizar la instrucción pública municipal, federalizándola, en detrimento de los poderes locales.

Al concluir el siglo XIX los reglamentos elaborados desde el ámbito sanitario y de la instrucción pública carecieron de una definición del edificio escolar. El Código Sanitario de 1891 y sus versiones reformadas de 1893 y 1903 consideraron las escuelas, que en su mayoría no se hallaban en edificios propios, sino en casas de alquiler, en el mismo rubro que los hoteles, mesones, casas de vecindad y dormitorios públicos. El capítulo "Habitaciones y escuelas" señalaba que las segundas estarían sujetas a la inspección sanitaria, que verificaría el saneamiento del terreno, las condiciones de los conductos desaguadores, los sanitarios o comunes y la provisión de agua potable, así como las leyes y reglamentos de instrucción pública (Consejo, 1891; 1903). Estos últimos, elaborados por la Secretaría de Justicia e Instrucción Pública, se limitaron a estipular de una manera inconsistente que el edificio escolar debía mantenerse aseado, ventilado "y con todas las demás condiciones que la Pedagogía, la Higiene y el decoro exigen en un establecimiento de esta naturaleza" ("Reglamento", 1891: 499 y 1897: 3-11). ${ }^{8}$ Pasaron varios lustros para que las prescripciones aprobadas por los congresos de higiene escolar e instrucción pública adquirieran una forma legal específica. ${ }^{9}$

En 1906 la Secretaría de Instrucción Pública y Bellas Artes, recientemente instituida y encabezada por el abogado Justo Sierra, publicó las condiciones que debían tener los locales

\footnotetext{
${ }^{8}$ El Reglamento Interior para las Escuelas Nacionales de Enseñanza Primaria de 1897 fue redactado con la colaboración del médico Luis E. Ruiz y el profesor Manuel Cervantes Imaz (Ruiz, 1900).

${ }^{9}$ La reglamentación francesa para la construcción de escuelas, que sirvió como referente para algunos arquitectos y pedagogos mexicanos, entre ellos Nicolás Mariscal y Abraham Castellanos, se encontraba vigente desde 1880 (Narjoux, 1881).
} 
adquiridos para la construcción o adaptación de edificios escolares en el Distrito Federal ("Condiciones", 1906-1907). Este reglamento técnico clasificó los edificios de acuerdo con el tipo de enseñanza al que estaban dirigidos y dictó, entre otras cosas, las condiciones para situar una escuela: el local debía ocupar un lote rectangular ubicado en el centro del pueblo o de la zona escolar, en un sitio elevado y un terreno permeable, a cien metros de distancia de lugares insalubres, peligrosos o ruidosos, en plazas o calles con obras de saneamiento, sin mucho tráfico vehicular y orientado hacia el sur, el oriente o el norte (véase cuadro 2).

Los edificios escolares de primera clase, por ejemplo, estaban preferentemente en una sola planta, con siete salones construidos y dos indicados en los planos (un vestidor para cada uno), dos salas-taller para trabajos manuales, biblioteca, sala de conferencias, vestíbulo, dirección con antesala, dos patios descubiertos para juegos y huertas, un patio cubierto para el gimnasio, lavabos, dos retretes y un mingitorio por cada sala de clase, un baño con regadera, privado de profesores y habitación del conserje. Una sala para clases albergaba a 48 alumnos en una superficie rectangular cuyas dimensiones oscilaban entre $6 \mathrm{~m}$ por $9 \mathrm{~m}$ y $7 \mathrm{~m}$ por $10 \mathrm{~m}$, con una altura máxima de $5 \mathrm{~m}$. La iluminación debía ser unilateral izquierda o bilateral diferencial con predominio del lado izquierdo de los alumnos, mientras que la ventilación se obtenía mediante ventanas de guillotina o un sistema que permitía abrir parte de ellas, además de dos puertas colocadas con hojas de madera en el lado opuesto (derecha). Los techos eran de vigueta de fierro, bóveda de ladrillo o cemento armado, sin cielos rasos, cornisas ni molduras, mientras que las paredes se pintaban con cal o colores de medio tono. El piso era de listones angostos de madera pintados al óleo, pero el de los pasillos y el resto de los departamentos estaba construido con cemento o mosaico. El servicio de agua potable era proporcionado mediante tuberías de fierro y las

\section{Cuadro 2}

\section{Clasificación de los edificios escolares según los reglamentos de 1906-1907}

\begin{tabular}{|c|c|c|c|c|}
\hline Edificio & Tipo de escuela & Superficie & $\begin{array}{l}\text { Número máximo de } \\
\text { alumnos }\end{array}$ & $\begin{array}{c}\text { Número de salas } \\
\text { para clases }\end{array}$ \\
\hline $1^{a}$ clase & $\begin{array}{l}\text { Primaria elemental y } \\
\text { superior }\end{array}$ & 2400 a $3000 m$ & 500 alumnos & 9 salas \\
\hline $2^{a}$ clase & Primaria superior & 1000 a $2000 m$ & 250 alumnos & 5 salas \\
\hline $3^{a}$ clase & $\begin{array}{l}\text { Primaria elemental de la } \\
\text { Ciudad de México y } \\
\text { cabeceras municipales }\end{array}$ & 1400 a $2000 \mathrm{~m}$ & 300 alumnos & 6 salas \\
\hline $4^{a}$ clase & $\begin{array}{l}\text { Primaria elemental de } \\
\text { las poblaciones rurales }\end{array}$ & 500 a $1000 m$ & No se especifica & 2 a 3 salas \\
\hline
\end{tabular}

Fuente: "Condiciones" (1906-1907). 
condiciones higiénicas de los inodoros debían ser las prescritas por el Consejo Superior de Salubridad ("Condiciones", 1907). ${ }^{10}$

Para la construcción de los nuevos edificios escolares, que debían sujetarse a las normas estipuladas en los reglamentos de 1906-1907, la administración de Porfirio Díaz recurrió al "contratismo," un modelo institucional para la provisión de obras públicas que consiste -según Priscilla Connolly- en que el gobierno contrata "la construcción de la obra a particulares, pero se encarga del suministro o comercialización del servicio" (Connolly, 1999: 147). Bajo este modelo, la Secretaría de Instrucción Pública encomendó en 1908, a través de la Junta Directiva de Obras de Edificios de Instrucción Primaria del Distrito Federal (creada en 1905 como un espacio de concertación de los actores involucrados en la construcción de escuelas),"1 la construcción de seis edificios escolares a la Compañía Bancaria de Obras y Bienes Raíces. Esta agrupación se especializaba en la especulación de bienes raíces y la creación de obras públicas, cuya mesa directiva estuvo conformada por reconocidos empresarios de la época: Fernando Pimentel, Leandro Payró, Luis Barroso Arias, Ramón Alcazar, Pablo Macedo, Jacinto Pimentel, Hugo Scherer Jr., Francisco Rincón Gallardo y Luis G. Fornel (Ruiz Zamudio, 2006) (véase cuadro 3).

\section{Cuadro 3}

\section{Edificios escolares encomendados a la Compañía Bancaria de Obras y Bienes Raíces, 1908}

\begin{tabular}{cl}
\hline Tipo de escuela & \\
\hline $1^{\mathrm{a}}$ clase & Esquina de las calles 6 ${ }^{\mathrm{a}}$ de las Artes y Manuel María Contreras \\
$1^{\mathrm{a}}$ clase & Ex Convento de las Bonitas \\
$1^{\mathrm{a}}$ clase & Ex Convento de las Bonitas \\
$3^{\mathrm{a}}$ clase & Avenida de la Paz \\
$3^{\text {a }}$ clase & Puente del Rosario \\
$3^{\text {a }}$ clase & $7^{\mathrm{a}}$ Calle del Cedro \\
\hline
\end{tabular}

Fuente: "Informe" (1908).

\footnotetext{
${ }^{10}$ Estos reglamentos, así como los resolutivos de los congresos de higiene e instrucción pública relativos al establecimiento de escuelas, codificaron una serie de conocimientos médicos, pedagógicos y de la construcción, vigentes o en proceso de emergencia. Asimismo, se basaban en las teorías miasmática y microbiana en salud pública, la pedagogía objetiva, la arquitectura racionalista y las técnicas de construcción tradicionales como la mampostería y otras modernas, como el concreto armado.

${ }^{11}$ La Junta estuvo conformada por ocho vocales nombrados por la Secretaría de Instrucción y uno por la Secretaría de Hacienda. En ella contaron con representantes las autoridades educativas (tres vocales) y hacendarias (un vocal), así como los arquitectos (tres vocales), los ingenieros (un vocal) y los propietarios de predios (un vocal), presididos por el Secretario de Instrucción Pública (Justo Sierra) o, en su ausencia, por el subsecretario del ramo (Ezequiel A. Chávez) ("Ley", 1905).
} 
Las escuelas construidas por la Compañía Bancaria de Obras constituían una cuarta parte de los edificios escolares que el gobierno federal llegó a poseer en la Ciudad de México para 1910 (véanse cuadros 3 y 4) y, a pesar de su reducido número, fueron utilizadas por la administración del general Díaz con un fin propagandístico: difundir los avances obtenidos en materia de instrucción pública en todo el país.

La escuela industrial La Corregidora de Querétaro ( $1^{a}$ Clase), construida al poniente de la Ciudad de México para impartir enseñanza técnica a las niñas, fue inaugurada en el mes de septiembre por el secretario Justo Sierra, como parte de la celebración del Centenario de la Independencia de México. Este acto se realizó en presencia de los representantes de las universidades de Pennsylvania, Yale y Texas (Crónica, 1991). En un folleto elaborado por

\section{Cuadro 4}

\section{Edificios escolares del gobierno federal en la Ciudad de México, 1910}

\begin{tabular}{|c|c|c|}
\hline Escuela & Situación & Costo \\
\hline Escuela Comercial Miguel Lerdo de Tejada & Esquina del Carmen y Santa Teresa & \\
\hline Escuela Industrial La Corregidora de Querétaro & Esquina de Villamil y Santa María la Redonda & $\$ 376987.52$ \\
\hline Escuela Industrial José María Chávez & Esquina de Aztecas y El Carmen & $\$ 144750.00$ \\
\hline Escuela Primaria Superior León Guzmán & Avenida de La Paz & $\$ 100548.95$ \\
\hline Escuela Primaria Superior Horacio Mann & Av. Chapultepec y Limantour & $\$ 116805.27$ \\
\hline Escuela Primaria Superior Florencio M. del Castillo & Esquina de Industria y Gómez Farías & $\$ 128130.97$ \\
\hline Escuela Primaria Superior Ignacio M. Altamirano & Esquina de Mina y Héroes & $\$ 232436.11$ \\
\hline Escuela Primaria Superior El Pensador Mexicano & $4^{a}$ del Ciprés & $\$ 128100.00$ \\
\hline Escuela Primaria Superior Padre Mier & Esquina de Artes y Manuel M. Contreras & $\$ 252264.64$ \\
\hline Escuela Primaria Elemental 330 & $7^{a}$ Calle del Cedro & $\$ 87173.32$ \\
\hline Escuela Primaria Elemental 270 & Calzada de Guadalupe & $\$ 23670.09$ \\
\hline Escuela Primaria Elemental 27 & Esquina Ribera de San Cosme y Norte 38 & $\$ 67191.51$ \\
\hline Escuela Primaria Elemental 272 & 3er Callejón de Rivero 3 & $\$ 9788.00$ \\
\hline Escuela Primaria Elemental 50 & ler Callejón de Rivero & $\$ 13314.72$ \\
\hline Escuela Primaria Elemental 9 & Jardín Alfonso Herrera & $\$ 26160.00$ \\
\hline Escuela Primaria Elemental 22 & Ex Garita de San Lázaro & $\$ 33363.60$ \\
\hline Escuela Primaria Elemental 61 & Ex Garita de Peralvillo & $\$ 12710.36$ \\
\hline Escuela Primaria Elemental 11 & Plazuela de la Santísima & $\$ 25763.27$ \\
\hline Escuela Primaria Elemental 37 & Calzada de Santa Crucita & $\$ 18268.20$ \\
\hline Escuela para niños tiñosos & $1^{\text {a }}$ de San Lorenzo 72 & $\$ 63700.00$ \\
\hline
\end{tabular}

Fuente: "Edificios" (1911). 
Juan Palacios y el médico Alfonso Pruneda el edificio fue presentado como "el palacio escolar más importante, en México, entre las escuelas primarias." Según la descripción del inmueble

[...] la obra obedeció a las prescripciones de la higiene escolar. Ocupa gran parte de una manzana, que fue asiento del antiguo convento de "Las Bonitas", totalmente demolido para levantar este edificio. Las salas son vastas, bien ventiladas y están vestidas de estuco; las paredes presentan superficies curvas en las esquinas para evitar que se deposite el polvo; los patios de los dos grandes departamentos que componen la escuela ocupan área muy considerable; las ventanas son muy amplias y tienen transparentes de colores propicios al trabajo escolar; hay un buen departamento de baños; la fachada vestida de hermosa cantería. El edificio todo es un modelo de propiedad, amplitud y belleza (Palacios y Pruneda, 1910: 27).

El edificio, que tuvo un costo superior al del resto de las construcciones escolares de la época, constaba de dos plantas, para recibir a 1296 alumnas, de acuerdo con los reglamentos que estipulaban un máximo de 48 alumnos por salón. En la planta baja se encontraban quince salones para clase, un baño, doce lavabos, dos WC, una bodega, una portería y las habitaciones del conserje. En la superior había doce salones para clase, un salón de actos, doce lavabos, sala para clase de cocina, dos lavaderos, proveeduría, comedor, subdirección, secretaría y la dirección de la escuela. Cabe destacar que La Corregidora de Querétaro no contó con superficies destinadas a biblioteca, huerto y gimnasio, como lo indicaban los requisitos para el establecimiento de una escuela de primera clase (véase imagen 1 ).

Al parecer, las compañías constructoras lograban imponer sus criterios sobre los parámetros de la higiene pedagógica. Así lo sugirió el médico inspector Manuel Uribe y Troncoso, quien al referirse a los edificios escolares construidos por la Compañía Bancaria de Obras y Bienes Raíces en la Plaza Villamil y en las calles Manuel M. Contreras, de las Artes y de La Paz, lamentó que el avance de las obras impidiera que se llevaran a cabo las modificaciones indicadas por los inspectores, "con lo cual quedaron sin corregir varios defectos higiénicos importantes" (1910: 186-187).12 A estas expresiones de insatisfacción se sumarían otras.

La construcción de "palacios escolares" como La Corregidora de Querétaro, a cargo de la Compañía Bancaria de Obras, fue objeto de severas críticas que se conjugaron con la crisis del

12 El diseño y la construcción de un edificio escolar fue objeto de disputas constantes que ponían en duda la efectividad de los lineamientos de la higiene escolar. Algunos médicos consideraban que los edificios debían seguir estrictamente las normas sanitarias impuestas por ellos en reglamentos y manuales de higiene escolar (De la Fuente, 1905; González, 1910). Sin embargo, para los profesores de instrucción primaria el papel de los médicos debía ser secundario, toda vez que la construcción de los edificios debía sujetarse a principios pedagógicos y arquitectónicos (Hernández, 1896; Castellanos, 1897). Los arquitectos e ingenieros, por su parte, estimaban que la aplicación de los principios higiénico-pedagógicos dependía, entre otros aspectos, de las condiciones climáticas y edafológicas de la zona del Distrito Federal donde se levantara el inmueble escolar. Esto se debía a que dichas condiciones los obligaban a hacer adecuaciones a los proyectos, sin desatender los reglamentos vigentes (Mariscal, 1906). 


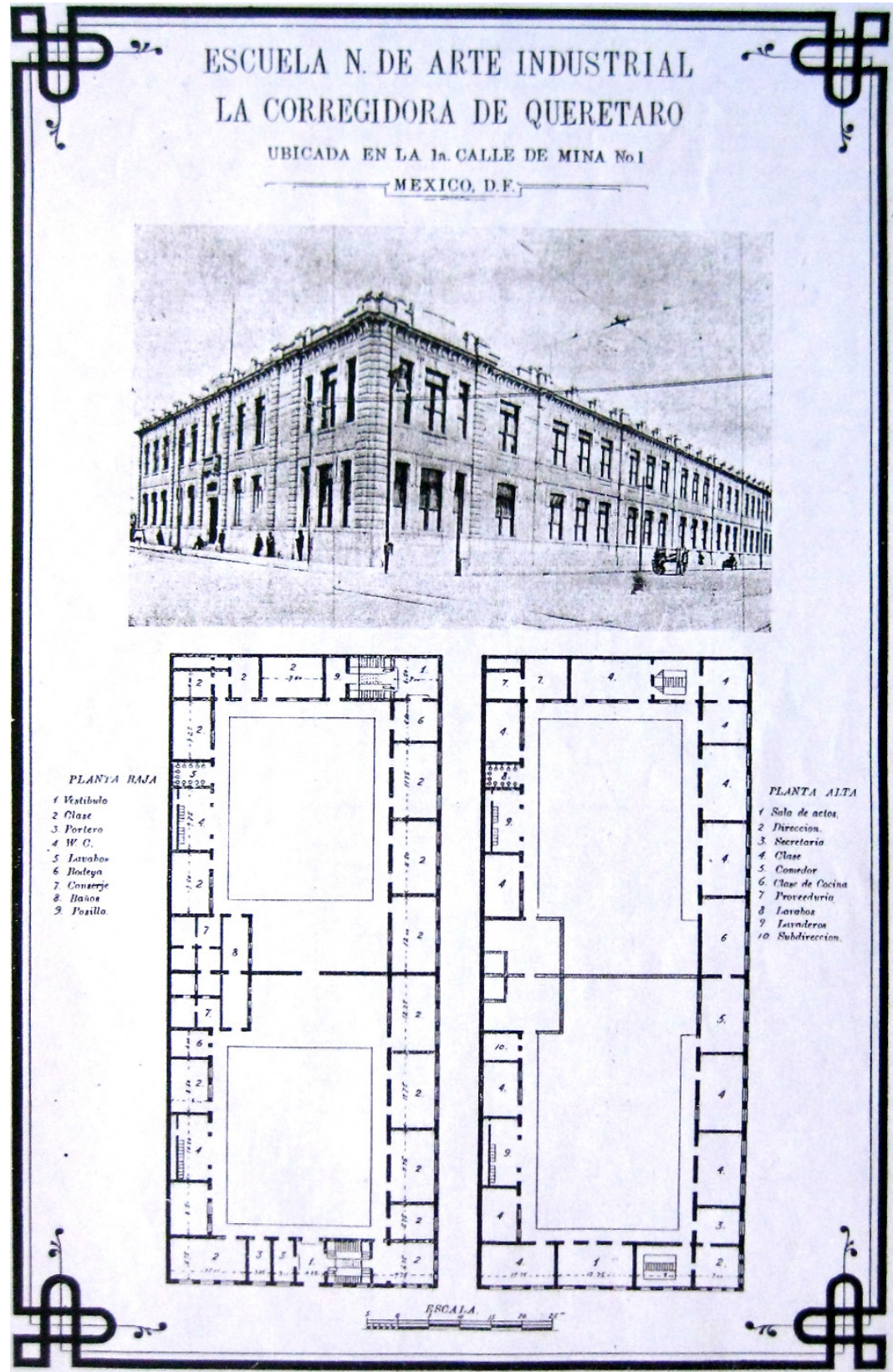

Imagen I. Escuela de Arte Industrial La Corregidora de Querétaro ( $1^{a}$ calle de Mina 1, México). Fuente: Álbum, 1916] 
sistema político porfiriano, al concluir la primera década del siglo xx. Lo que para algunos actores técnicos, como el arquitecto-ingeniero Manuel Francisco Álvarez (1910), significó un triunfo del gobierno porfiriano en materia educativa, para otros, como los ingenieros Félix Palavicini (1908) y Alberto Pani (1912; 1916), fue una política irracional que privilegió la construcción de costosos templos escolares en detrimento de la cobertura educativa para las clases populares, cuyo nivel de analfabetismo consideraban como alarmante.

La inconformidad hacia este tipo de políticas porfirianas condujo a varios actores a optar por la organización de un nuevo sistema político que diera cabida a sus proyectos técnicos, como los relativos a la construcción económica de escuelas. Finalmente la revolución constitucionalista, a la que se adhirió un número importante de técnicos ocupado en diseñar un programa de reformas político-sociales para el nuevo gobierno, reconoció, por primera vez, la existencia de una arquitectura escolar mexicana que sus promotores desearon replicar para todo el país, de acuerdo con la referida imagen de la Ciudad de México como foco irradiador de la cultura moderna.

En 1916 la Secretaría de Instrucción Pública y Bellas Artes, bajo el encargo de Félix Palavicini, publicó una "cartilla de arquitectura escolar nacional" que contenía un nuevo código técnico en el cual se proscribió el uso y adaptación de casas como escuelas, sobre las bases del conocimiento médico-pedagógico. Además, incluyó los planos y fachadas de las escuelas del Distrito Federal que podrían servir como prototipo a las que se construyeran en el futuro en todo el país (Álbum, 1916). Las imágenes correspondían a los palacios escolares construidos durante la última década porfiriana, incluido el inmueble de La Corregidora de Querétaro, pero obedeciendo a una nueva orientación política: la educación de las clases populares, que sirvió como uno de los pilares ideológicos para consolidar al sistema político posrevolucionario. Se trató, en suma, de reorientar políticamente a la arquitectura escolar erigida en el Porfiriato.

\section{Conclusión}

Tanto los reglamentos técnicos sobre el establecimiento de escuelas, particularmente aquellos publicados en 1906, 1907 y 1916, como la construcción de edificios escolares tipo -por ejemplo, el de La Corregidora de Querétaro-, fueron finalmente reconocidos como la simiente de una arquitectura escolar nacional. Ambos constituyen el resultado de los conocimientos técnicos relativos a la salud, la enseñanza y la construcción, así como de las acciones emprendidas por sus promotores y las decisiones adoptadas por el poder público, que favoreció la erección de templos, palacios o monumentos escolares, como se les llegó a denominar en 
la época. La interacción entre factores epistémicos y no epistémicos conformó el acto técnico que en este artículo hemos Ilamado arquitectura escolar.

El estudio de la arquitectura escolar de la Ciudad de México requirió la elaboración de una propuesta metodológica complementaria, que se nutriera del trabajo realizado desde varias perspectivas historiográficas de la educación, la salud y la arquitectura, sobre todo de aquellas que reconocieron la dimensión material de la cultura escolar y la dimensión socio-política de las obras materiales. La Historia Política de la Tecnología nos proporcionó un conjunto de categorías tales como acto técnico, actores técnicos y sistema político, que nos permitieron concebir el edificio escolar como resultado de la interacción del conocimiento con la política.

No obstante, para comprender la complejidad de este acto técnico, es necesario emprender nuevos estudios sobre otras arquitecturas escolares (no sólo la de carácter monumental), en distintos periodos históricos y más allá de los efectuados en la Ciudad de México. Es importante, también, que estudios posteriores aborden la percepción pública del acto técnico denominado como arquitectura escolar, especialmente la población escolar y la comunidad donde se erigieron los nuevos edificios, así como las acciones a que pudo dar lugar dicha percepción, tanto en el diseño como en el uso de los inmuebles. De esta forma, los estudios sobre la arquitectura y el espacio escolar son necesariamente complementarios -cada uno con sus particularidades metodológicas-, para conocer la dimensión material de la escuela.

\section{Fuentes}

\section{Archivos}

Archivo Histórico del Distrito Federal (AHDF), Fondo: Ayuntamiento

Archivo Histórico de la Secretaría de Salud (AHSS), Fondo: Salubridad Pública

Biblioteca Nacional de México (BNM), Fondo Reservado

\section{Fuentes Primarias}

"Actas de las sesiones del Congreso Higiénico Pedagógico" (1882), Archivo Histórico de la Secretaría de Salud, Fondo Salubridad Pública, Sección Congresos y convenciones, caja l, expediente 5. Álbum escolar de México (1916), México, Sección de Construcción y Reparación de Escuelas.

Álvarez, Manuel Francisco (1910), Les édifices d'instruction publique à Mexico et l'état d'avancement réalisé dans les établissments officiels et particuliers, jusqu'en 1909. Ille Congrès Internacional d'Hygiène Scolaire, México, Tipografía Económica.

Castellanos, Abraham (1897), Organización escolar (Ensayo crítico) por Abraham Castellanos, Oaxaca, Imprenta de Lorenzo San Germán.

Código sanitario de los Estados Unidos Mexicanos, México, Secretaría de Gobernación, Imprenta de la Patria de I. Paz. [documento pdf disponible en: <https://archive.org/stream/cdigosanitariod 00mexigoog\#page/n6/mode/2up> (consulta: 29/9/15).] 
"Condiciones que deberán tener los locales que se adquieran para la construcción o adaptación de Edificios Escolares en el Distrito Federal" (1906-1907), Boletín de Instrucción Pública, t. VI, núm. 1, pp. 40-42.

"Condiciones que deberán tener los Edificios Escolares de Primera Clase, para alojar Escuelas de Instrucción Primaria Elemental y Superior" (1907), Boletín de Instrucción Pública, t. VII, núm. 1, pp. 221224.

"Congreso Nacional de Instrucción" (1890), La Escuela Moderna, vol. I, núm. 13.

Consejo Superior de Salubridad (1891), Código Sanitario de los Estados Unidos Mexicanos, México, Imprenta de "La Patria".

Consejo Superior de Salubridad (1903), Código Sanitario de los Estados Unidos Mexicanos, México, Imprenta de Eduardo Dublán.

Crónica oficial de las fiestas del primer centenario de la Independencia de México, publicado bajo la dirección de Genaro García (1991), México, Centro de Estudios de Historia de México.

"Dictamen sobre locales para escuelas" (1890), La Escuela Moderna, vol. I, núm. 12.

"Edificios de propiedad Federal ocupados por Escuelas Primarias en el Distrito Federal, con indicación del costo de alguna de ellas" (1911), Boletín de Instrucción Pública, t. XVI, núm. 1-2, pp. 26-30.

Fuente, José M. de la (1905), Elementos de Higiene Pedagógica por el Dr. José M. de la Fuente, México, Imprenta del Gobierno Federal.

González, José de Jesús (1910), Higiene escolar, $1^{a}$ ed., México, s. p. i.

(1918) Higiene escolar, $2^{a}$ ed., México, Librería de la Vda. De Ch. Bouret.

Hernández, Julio S. (1896), Álbum pedagógico y escolar: conferencias científicas dadas en la Escuela Normal de México, México, Secretaría de Fomento.

"Informe relativo a las labores de la Junta Directiva de Edificios de Instrucción Primaria del Distrito Federal, del 30 de noviembre de 1905 al 30 de junio de 1908" (1908), Boletín de Instrucción Pública, t. X, núm. 3-4, pp. 460-469.

"Ley reglamentaria para la constitución y funciones de la Junta Directiva de Obras de Edificios de Instrucción Primaria del Distrito Federal” (1905), Boletín de Instrucción Pública, t. V, núm. 30, pp. 245-246.

Mariscal, Nicolás (1906), "Concurso para edificios escolares," El Arte y la Ciencia, vol. VII, núm. 9, pp. 230-235.

Martínez, Faustino (1891), "Bases para la construcción de edificios para escuelas municipales", Archivo Histórico del Distrito Federal, Fondo Ayuntamiento. Sección de Instrucción Pública, vol. 2505, exp. 2660.

Narjoux, Felix (1881), Les écoles publiques, construction et installation en France et en Anglaterre, Paris, Ve A. Morel.

Palacios, Juan y Alfonso Pruneda (1910), Guía de Instrucción Pública y Bellas Artes, México, Imprenta de Manuel León Sánchez.

Palavicini, Félix (1908) [1924], "La construcción económica de escuelas", en Marcos Becerra, Palavicini desde allá abajo. Historia del hombre, pedagogo, político, ladrón, diplomático, periodista, ciudadano, México, Talleres Linotipográficos de "El Hogar".

Pani, Alberto (1912), La instrucción rudimentaria en la República, México, Müller Hermanos. (1916), La Higiene en México, México, Imprenta de Ballescá.

Pérez Gallardo, Rafael (1883), La Instrucción Pública Municipal en la Ciudad de México en 1882, México, Tipografía Literaria de F. Mata.

"Reglamento interior de las escuelas oficiales de enseñanza primaria elemental (1891), citado por Meneses Morales, Ernesto (1998), Tendencias educativas oficiales en México: 1821-1911, México, Universidad Iberoamericana. 
"Reglamento Interior para las Escuelas Nacionales de Enseñanza Primaria" (1897), Revista de la Instrucción Pública, t. II, núm. 1, pp. 3-11.

Ruiz, Luis E. (1900), Tratado elemental de pedagogía, México, Oficina Tipográfica de la Secretaría de Fomento

Uribe y Troncoso, Manuel (1910), "Informe de los trabajos ejecutados por el Servicio Higiénico Escolar, desde el $1^{\circ}$ de julio de 1908 hasta el 31 de julio de 1909", Boletín de Instrucción Pública, t. XV, núm. 1-6, pp. 186-187.

\section{Fuentes secundarias}

Arañó, Axel (2011), Arquitectura Escolar. SEP 90 años, México, SEP-Conaculta.

Arias Montes, J. Víctor (2005), Juan O’Gorman. Arquitectura Escolar 1932, México, UAM-UNAM-UASLP (Raíces 4. Documentos para la historia de la arquitectura mexicana).

Bazant, Mílada (2000), "La creación de la élite profesionista", en Mílada Bazant, Historia de la educación durante en Porfiriato, México, El Colegio de México, pp. 217- 260.

Bencostta, Marcus Levy (2005), História da Educaçao, arquitetura e espaço escolar, São Paulo, Cortez Editora.

Bencostta, Marcus Levy y Rosa Fátima de Souza (eds.) (2013), "Dossié: Cultura Material Escolar: abordagens históricas", Educar em Revista, núm. 49.

Brandariz Gustavo (1997), La arquitectura escolar de inspiración sarmientina, Buenos Aires, Universidad de Buenos Aires.

Carrillo, Ana María (1999), "El inicio de la higiene escolar en México: Congreso Higiénico Pedagógico de 1882", Revista Mexicana de Pediatría, vol. 66, núm. 2, pp. 71-74.

(2005), "Vigilancia y control del cuerpo de los niños. La Inspección Médica Escolar (1896-1913)", en Laura Cházaro y Rosalina Estrada (coords.), En el umbral de los cuerpos. Estudios de antropología e historia, Michoacán, El Colegio de Michoacán-BUAP, pp. 171-207.

(2010), Epidemias, saber médico y salud pública en el Porfiriato, 2 vol., tesis de doctorado en Historia, México, Facultad de Filosofía y Letras/UnAM.

Chaoul Pereyra, María Eugenia (2005), "La escuela nacional elemental en la Ciudad de México como lugar, 1896-1910", Secuencia, núm. 61, pp. 144-176.

Châtelet, Anne-Marie (2004), "Essai d'historiographie I. L'architecture des écoles aux XXe siècle", Histoire de l'education, núm. 102, pp. 7-37.

Connolly, Priscilla (1999), "Obras públicas", en Sandra Kuntz Ficker y Priscilla Connolly (coords.), Ferrocarriles y obras públicas, Instituto Mora-El Colegio de Michoacán-El Colegio de México-Instituto de Investigaciones Históricas/UnAM, pp. 150-151.

Donzelot, Jacques (2008), La policía de las familias. Familia, sociedad y poder, Buenos Aires, Ediciones Nueva Visión.

Espinoza, Lucía (2005), Polis científica: arquitectura escolar y Estado moderno. Santa Fe 1900-1943, Santa Fe, Universidad Nacional del Litoral.

Estadísticas sociales del Porfiriato 1877-1910 (1956), México, Dirección General de Estadística/Secretaría de Economía.

Fernández Christlieb, Federico (2000), Europa y el urbanismo neoclásico en la Ciudad de México. Antecedentes y esplendores, México, Instituto de Geografía/unam.

Fisher, Jaime (2010), El hombre y la técnica. Hacia una filosofía política de la ciencia y la tecnología. México, Coordinación de Humanidades/UnAM.

Gama, Ruy (1985), "Palavras e palavras: Temas para una história da tecnología", Quipu, Revista Latinoamericana de Historia de las Ciencias y la Tecnología, vol. 2, núm. 1, pp. 87-93.

Julien, Marie-Pierre y Céline Rosselin (2005), La Culture Matérielle, París, La Découverte, (Repères 431). 
Kuntz Ficker, Sandra y Priscilla Connolly (coords.) (1999), Ferrocarriles y obras públicas, Instituto Mora-El Colegio de Michoacán-El Colegio de México-Instituto de Investigaciones Históricas/UnAM.

López Oresta, Norma Ramos y Armando René Espinosa (2013), "La intervención del Estado, la desigualdad y el deseo en la invención de los espacios escolares rurales en tres regiones de México en el periodo posrevolucionario", Educar em Revista, núm. 49, pp. 59-82.

Maldonado Tapia, Rafael (1999), Historia de la arquitectura escolar en Colombia, Bogotá, Universidad Nacional de Colombia.

Medina Peña, Luis (2004), La invención del sistema político mexicano. Forma de gobierno y gobernabilidad en México en el siglo xIx, México, Fondo de Cultura Económica.

Mendes de Faria Filho, Luciano (1998), "O espaço escolar como objeto da história da educação: algunas reflexões", Revista da Faculdade de Educação, vol. 24, núm.1, pp. 141-159.

Meníndez Martínez, Rosalía (2013), Las escuelas primarias de la Ciudad de México en la modernidad porfiriana, México, Universidad Pedagógica Nacional.

Ortega y Gasset, José (1965), Meditación de la técnica, España, Espasa-Calpe.

Ramírez Potes, Francisco (2009), "La arquitectura escolar en la construcción de una arquitectura del lugar en Colombia", Revista Educación y Pedagogía, vol. 21, núm. 54, pp. 81-101.

Rodríguez Kuri, Ariel (1999), "Gobierno local y empresas de servicios: la experiencia de la Ciudad de México en el Porfiriato", en Sandra Kuntz y Priscilla Connolly (coords.), Ferrocarriles y obras públicas, México, Instituto Mora-Colmich-Colmex-IIH/UnAM, pp. 165-190.

Rojas Rabiela, Teresa, José Luis Martínez y Daniel Murillo Licea (2009), Cultura hidráulica y simbolismo mesoamericano del agua en el México prehispánico, Centro de Investigaciones y Estudios Superiores en Antropología Social (CIESAS)-Instituto Mexicano de Tecnología del Agua (IMTA).

Rosa, Natalia de la (2011), "La pragmática arquitectónica, 1924-1929. Construcción escolar en el régimen callista", en Axel Arañó, Arquitectura Escolar. SEP 90 años, México, Conaculta/SEP.

Ruiz Zamudio, Eunice (2006), Burguesía empresarial, 1880-1915: un empresario mexicano: el caso de Luis Barroso Arias y socios, tesis de licenciatura en Historia, UAM-Iztapalapa, México.

Saldaña, Juan José (2011), Conocimiento y acción. Relaciones históricas de la ciencia, la tecnología y la sociedad en México, México, Plaza y Valdés-Facultad de Filosofía y Letras/Unam

Sánchez Ruiz, Gerardo (2005), "Las condiciones sociales que exigieron la opción técnica de las escuelas Bassols-O'Gorman", en J. Víctor Arias Montes (coord.), Juan O'Gorman. Arquitectura Escolar 1932, México, UAM Azcapotzalco-UNAM-UASLP.

Schávelzon, Daniel (1989), "Sarmiento y la Escuela Modelo de Catedral al Norte (1860)", Summa-Temática (Arquitectura para la Educación), núm. 33, pp. 18-23.

Souza, Rosa Fátima de (1998), Templos de Civilização: a implantação da escolar primária graduada no Estado de São Paulo, São Paulo, Editora UNESP Fundação.

Staples, Anne (2008), "Primeros pasos de la higiene escolar decimonónica", en Claudia Agostoni (coord.), Curar, sanar y educar. Enfermedad y sociedad en México, siglos XIX y xx, México, UNAM-BUAP, pp. 17-42. Vargas Salguero, Ramón (1998), Afirmación del nacionalismo y la modernidad, vol. III, t. II, en Carlos Chanfón Olmos (coord.), Historia de la arquitectura y el urbanismo mexicanos, FCE-UNAM.

Carlos Ortega Ibarra. Doctor en Historia por la Facultad de Filosofía y Letras de la UNAM. Investigador docente en la Universidad Pedagógica Nacional, Unidad 151, Toluca. Líneas de investigación: Historia de la tecnología en México e Historia de la arquitectura escolar. Publicaciones recientes: Ortega Ibarra, Carlos (2012), "Ciencia y revolución en la 
arquitectura escolar. Ciudad de México (1910-1920)", en Ciencia y desarrollo, vol. 38, núm. 259, pp. 39-42. Ortega Ibarra, Carlos y Juan José Saldaña, (2013), "Primeros reglamentos técnicos en arquitectura escolar en México", en Quipu. Revista Latinoamericana de Historia de las Ciencias y la Tecnología, vol. 15, núm. 1, pp. 65-84.

Recibido: 9 de diciembre de 2014

Aceptado: 13 de febrero de 2015 